\title{
Human Factors and Peri-operative Safety: Compliance with Five Steps to Safer Surgery (5STSS) Protocols at an NHS Hospital during the SARS-CoV2 Pandemic1 - Human Factors Implications
}

\author{
Dr Asekhame Oise Isemede *, Dr Sarah O. Beckley \\ Department of Anaesthetics, St. Richard's Hospital, Chichester, PO19 6SE, England, United Kingdom \\ *Corresponding Author: Dr Asekhame Oise Isemede; aisemede@yahoo.com
}

Received 12 January 2021;

Accepted 29 January 2021;

Published 01 February 2021

\begin{abstract}
The five steps to safer surgery processes are essential standards for the delivery of safe surgery. The steps are Team Brief, World Health Organization (WHO) - Sign In, Time Out, Sign Out and Team Debrief. With increased anxieties and other disruptive human factors associated with the COVID-19 pandemic capable of negatively affecting care standards, this audit of compliance with the five steps to safer surgery was done to determine if peri-operative safety standards have been maintained or not during the COVID-19 pandemic1 at the Trust. Methods: A retrospective audit of completeness of documentations of the above five steps (used as proxy for compliance) obtained from electronic medical records of a sample of patients who had surgical procedures during the COVID-19 pandemic1 (Part A) and a survey of peri-operative staff on COVID-19 and peri-operative care (Part B) were carried out after approval by the audit department of the Trust. Results: Compliance with Team Briefs, WHO - Sign In, Time Out and Sign Out were comparable to 2019 audit and national standards but there was a fall in compliance with Team Debrief from 100\% (pre-COVID-19 audit) to 52\%. Fear of getting infected with COVID-19 and lack of suitable care guidelines were found to be potent anxiety factors for the majority of respondents, all the respondents supported a review of the current peri-operative care pathway. Conclusion: All causes including human factors contributing directly or indirectly to the fall in standards seen in this audit need to be explored and strategies (solutions) such as development of new peri-operative care pathways capable of improving team resilience for more effective and safer peri-operative care should be urgently considered.
\end{abstract}

Keywords: COVID-19 pandemic, peri-operative safety.

\section{Introduction}

Unsafe surgical care is a serious challenge affecting all countries with major complication rates ranging from 3\%-16\% and death rates ranging from $0.2 \%$ to $10 \%$ annually; the result is at least 7 million disabling complications and one million deaths each year (WHO, 2000). In response to this, the World Health Organization (WHO) launched a global patient safety initiative; 'Safe Surgery Saves Lives' (WHO 2008). The Safe Surgery Saves Lives programme involved trial of a checklist by surgical teams which demonstrated a $36 \%$ reduction in post-operative complications (Haynes et al, 2009). Consequently, the NPSA made the checklist a national requirement for hospitals in the UK (NPSA 2009, NHS Institute for Innovation and Improvement 2009).

Furthermore, Leonard et al (2004) and Einav et al (2010) demonstrated significant contribution of poor communication among surgical teams to unsafe surgical care, leading to the development of briefings or team huddles. The aim of the team huddle (safety huddle or team brief) is to help create a broader knowledge base for planned surgical lists so that each team member has a better understanding of the tasks at hand, and can anticipate future events and plan accordingly. Lingard et al (2011) showed improved clinical practice in participating teams with the introduction of the team huddle. Consequently, pre-operative and post-operative surgical safety huddle aimed at improving team communication among surgical teams was developed and together with the three steps of the WHO surgical safety checklist (WHO Sign In, Time Out and Sign Out), the five steps to safer surgery emerged as essential safety standards for effective team communication, team working and team leadership for the delivery of safe surgery applicable to all operative theatre environments at all times (NPSA 2010, Vickers, 2011).

The five steps to safer surgery are easy to use for both simple and complex surgical procedures and has contributed to safer surgery in the last decade by improving team communication and team working among the many professions involved in 
surgical care, it has reduced adverse events and improved the quality and safety of surgical services (Patel et al 2014, Haynes et al 2009, Treadwell et al 2014). Team Briefings and Debriefings also help with better identification of recurring issues and a reduction in unexpected delays (Jain et al 2015, Allard et al 2011, Papaspyros et al 2010) as well as improvements in situational awareness and earlier recognition of patients at risk of postoperative deterioration (Brady and Goldenhar 2014). In 2017, the Care Quality Commission (CQC), England reported a potential link between reductions in never events and increased use of the five steps to safer surgery checklists in some NHS trusts (CQC, 2017).

With the five steps to safer surgery established as essential peri-operative safety standard for effective team communication, team working and team leadership for the delivery of safe surgery applicable to all operative theatre environments at all times (NPSA 2010, Vickers, 2011), this study was developed with the following aims: First, to demonstrate if peri-operative safety standards with respect to the five steps to safer surgery processes were maintained or not during the COVID-19 pandemic1, second, as a support for continuous quality improvement through research, service evaluations and audits called for by Professor Chris Whitty the CMO and NIHR Co-Lead (Whitty, 2020) as national and most local quality assurance processes were put on hold because of the pandemic and third, to explore, analyse and proffer solutions for causes including human factors that may be identified for any significant deviation from standards and make recommendations to meet required standards (Healthcare Commission, 2004).

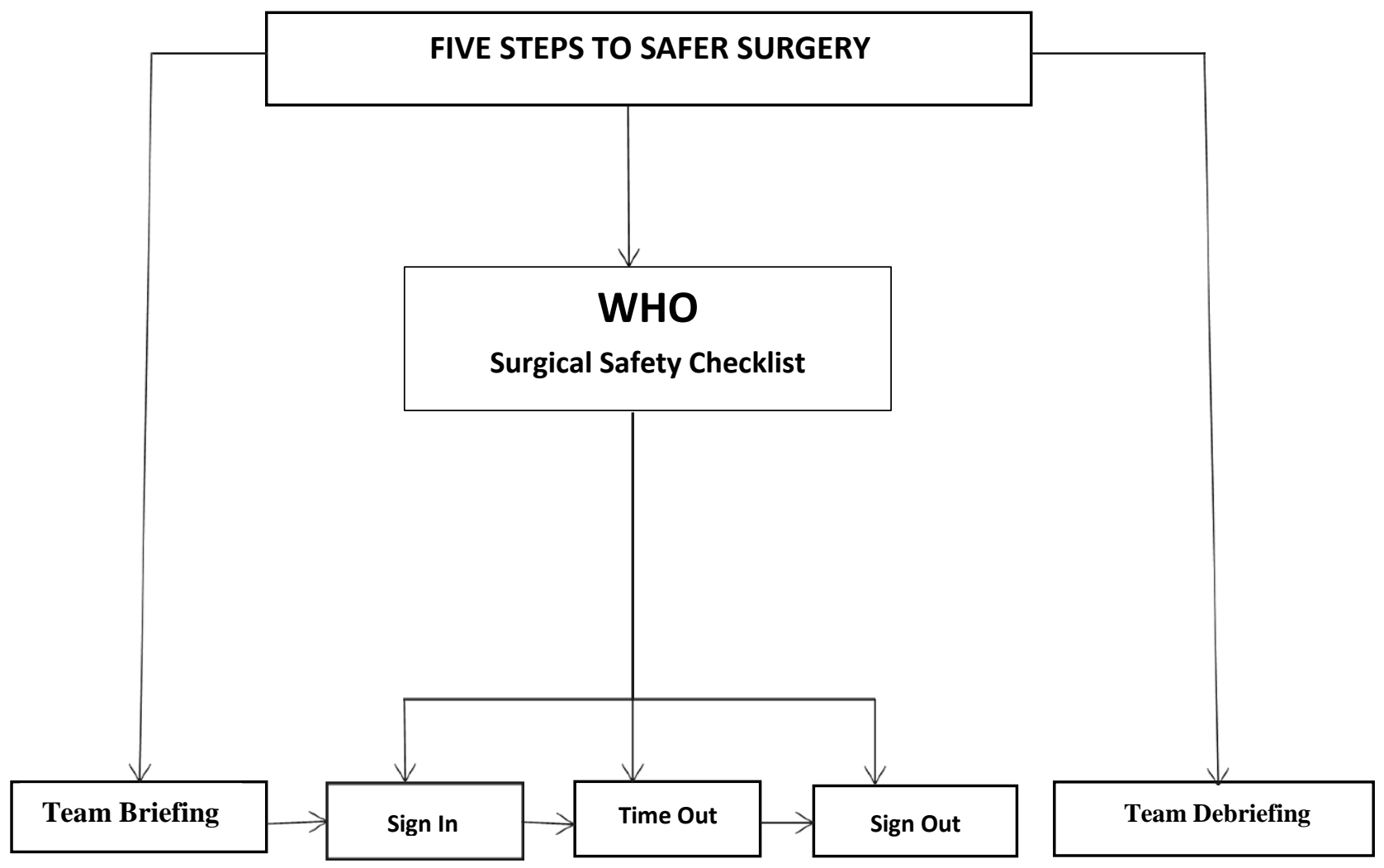

(Safe Surgery Saves Lives Initiative: WHO 2008, Haynes et al. 2009, NPSA 2009, NPSA 2010, and Vickers 2011)

\section{Methods}

A retrospective cross sectional observational audit using routinely collected patients' care data on electronic medical records accessed after formal approval from trust audit department. All cases in the second week of May and the third week of June, 2020 (covid-19 pandemic1) were analysed (non-probability sample).

A survey of staff on anxiety inducing factors at work during the COVID-19 pandemic and potential strategies for mitigation was also carried out (Part B), this was an anonymous survey and all Operating theatres staff were invited to participate.

Compliance data were analysed using RAG (red, amber and green) rating - red being less than 90\% compliance, amber between $90 \%$ and $94 \%$ compliance and green $95 \%$ and above, these were compared with 2019 (pre pandemic) compliance levels.

Ethical approval was obtained through a formal application process to the clinical audit department of the Trust, this audit was approved (Audit registration number 923) and access to electronic patients' records was granted (for this audit only).
Methodological quality was ensured through a 2 persons (a colleague and I) approach to data collection and verification to maximise the reliability of this audit.

\section{Results}

Part A: A total of 124 surgical procedures were completed during the study period, of these 124 cases, 113 World Health Organization (WHO) forms were available on the electronic computer document management (ECDM) system, representing (91\%), 110 of these 113 forms (97.3\%) were fully completed (compliant).

Team Briefs: 62 teams managed the 124 cases on 62 operating lists, and out of a possible total of 62 Team Brief forms, 50 were located (80\%), and all 50 forms (100\%) were fully completed (compliant) for Team Briefing but only 26 out of the 50 forms (52\%) were fully completed (compliant) for team debriefing.

Part B: Survey of peri-operative staff on issues arising from managing COVID-19 surgical procedures during the pandemic. 
Thirty questionnaires were returned out of forty distributed (75\%) return rate). Fear of contracting Covid-19 infection $(+4$ on a weighting scale of -5 to +5$)$, lack of adequate care guidelines to safely manage both COVID-19 and non-COVID-19 cases (+3.5), difficulties with vision and hearing when donned with PPEs $(+3.2)$ and work intensity/fatigue $(+3.1)$ had high scores as anxiety provoking factors for respondents while cancellations of holidays (4.4), lack of PPEs (- 4.0) were reported as non-anxiety provoking factors by respondents.

All 30 respondents welcomed a review of the current perioperative care pathway, 2 respondents $(6.6 \%)$ opted for a review of WHO SSCL only while 13 respondents (43.3\%) opted for a review of Team Brief and Team Debrief only and 14 respondents (46.6\%) opted for a review of WHO SSCL, Team Brief, Team Debrief and amalgamation of the forms into a single form.

\section{Results}

Table1: Compliance with World Health Organization Surgical Safety Checklist (WHO SSCL).

\begin{tabular}{|l|l|l|l|l|}
\hline No. of Procedures & Available on ECDM & Fully Completed & Compliance with WHO SS CL & Compliance 2019 Audit \\
\hline 124 & $113(91 \%)$ & 110 & $97.3 \%$ & $100 \%$ \\
\hline
\end{tabular}

ECDM: Electronic computer document management system

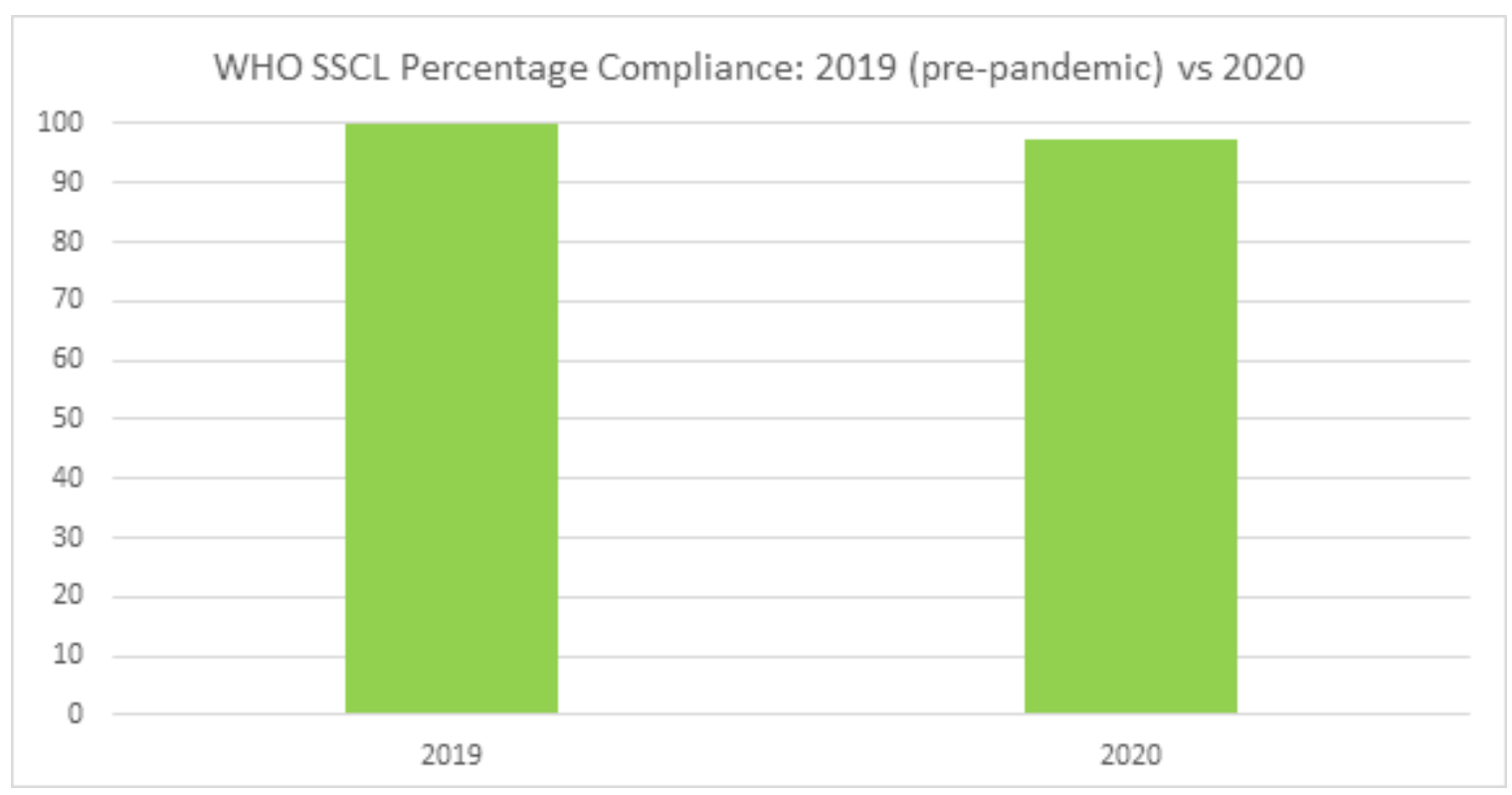

Figure 1: Compliance with WHO SSCL: Pre-pandemic (2019) vs 2020

Table 2a: Compliance with Team Brief (TBrief)

\begin{tabular}{|l|l|l|l|l|}
\hline No. of Procedures & Lists/ Teams & Forms available & Compliance with TBrief & Compliance with TBrief (2019 Audit) \\
\hline 124 & 62 & $50 / 62(80 \%)$ & $50 / 50(100 \%)$ & $(100 \%)$ \\
\hline
\end{tabular}

Table 2b: Compliance with Team Debrief (TDBrief).

\begin{tabular}{|l|l|l|l|l|}
\hline No. of Procedures & Lists/ Teams & Forms available & Compliance with TDbrief & Compliance with TD brief 2019 Audit \\
\hline 124 & 62 & $50 / 62(80 \%)$ & $26 / 50(52.0 \%)$ & $(100 \%)$ \\
\hline
\end{tabular}

\section{COMPLIANCE WITH TEAM BRIEF AND TEAM DEBRIEF: PRE- PANDEMIC (2019 VS 2020 AUDIT}

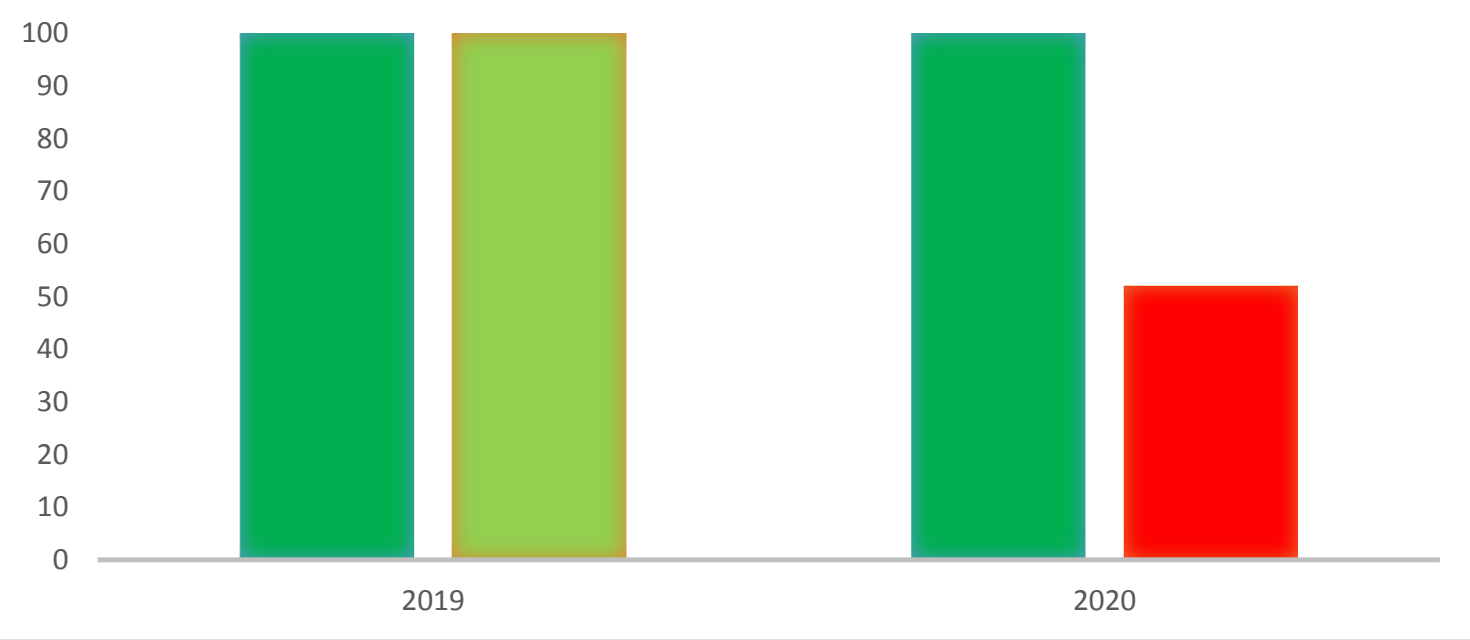

Figures 2a and 2b 
Table 3: COVID-19 and Healthcare: anxiety inducing factors

\begin{tabular}{|l|l|l|l|l|l|l|l|l|l|l|l|l|}
\hline Anxiety inducing factors & A & B & C & D & E & F & G & H & I & J & K & L \\
\hline Number of respondents & 30 & 30 & 30 & 28 & 29 & 29 & 29 & 30 & 29 & 30 & 29 & 30 \\
\hline Cumulative weight (CWT) & +120 & -120 & +105 & +28 & -116 & -35 & +75 & +40 & +90 & -132 & +88 & +98 \\
\hline Average Weight (AWT) & +4 & -4 & +3.5 & +1 & -4 & -1.2 & +2.5 & +1.3 & +3.1 & -4.4 & +3.0 & +3.2 \\
\hline Ranking & 1 & 11 & 2 & 8 & 10 & 9 & $\mathbf{6}$ & 7 & 4 & 12 & 5 & 3 \\
\hline
\end{tabular}

Weight (-5 to +5): range from -5: strongly disagree, through Zero (0): nether agree nor disagree to 5: strongly agree), Cumulative Weight (CWT)

$=$ Sum of weights allocated by respondents (Anxiety severity on $\mathrm{X}$ axis)

Anxiety Inducing Factors as reported by peri-operative staff:

A = Fear of "catching Covid-19",

$\mathrm{B}=$ Inadequate or lack of PPEs and other equipment,

$\mathrm{C}=\mathrm{Lack}$ or unclear guidelines for patient care,

$\mathrm{D}=$ Blame culture,

$\mathrm{E}=$ Inadequate psychological support and staff wellbeing information from organisation,

$\mathrm{F}=$ Lack of understanding of personal stress by managers and organisation,

$\mathrm{G}=$ Worries about when to isolate,

$\mathrm{H}=$ Concerns about cancellations of care for non-COVID patients,

$\mathrm{I}=$ Increased workload (Fatigue),

$\mathrm{J}=$ Cancellation of holidays,

$\mathrm{K}=$ Physical injuries ("COVID battle scars") from wearing PPEs,

$\mathrm{L}=$ Difficulties with vision and hearing when wearing PPEs

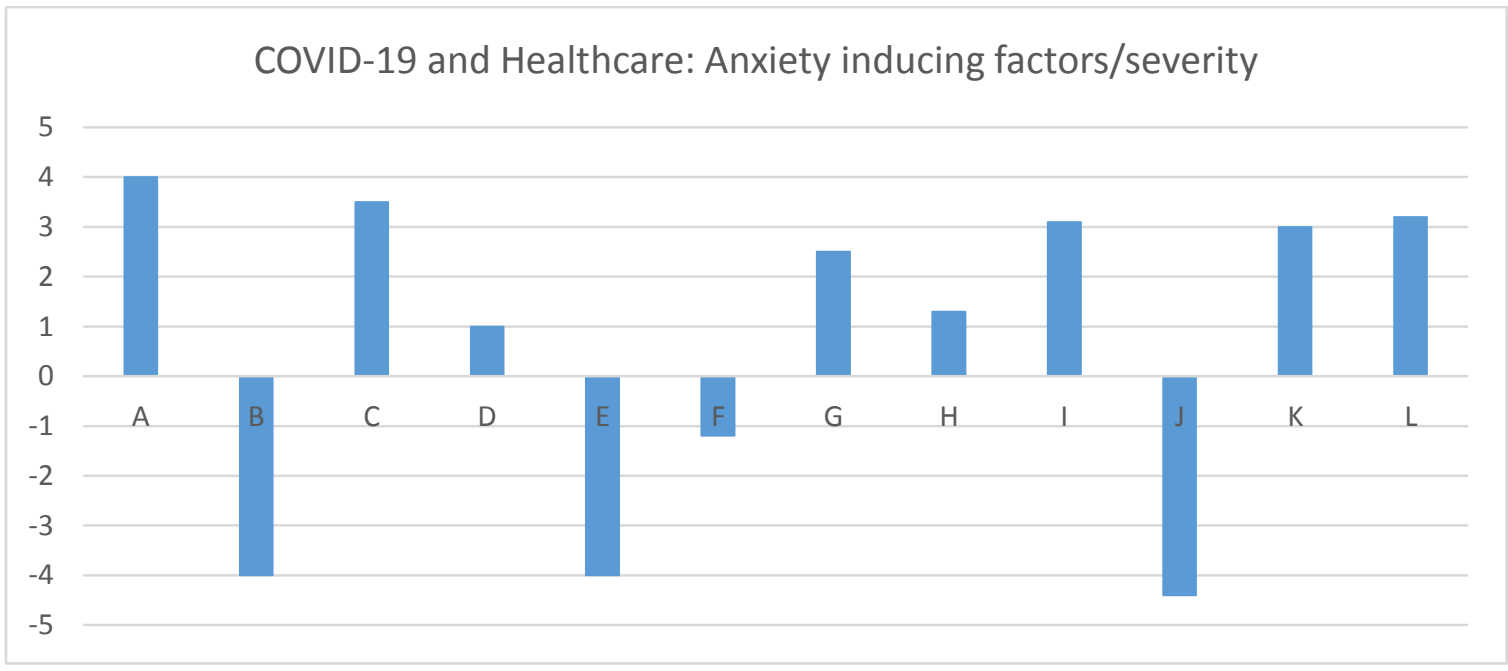

Figure 3: COVID-19 and Healthcare: anxiety inducing factors

Table 4: Perioperative safety pathway review: preferences of respondents

\begin{tabular}{|l|l|l|l|}
\hline Review of: & $\begin{array}{l}\text { WHO SSCL } \\
\text { only }\end{array}$ & $\begin{array}{l}\text { Team Brief and Team } \\
\text { Debrief only }\end{array}$ & $\begin{array}{l}\text { WHO SSCL, Team Brief, Team Debrief and } \\
\text { amalgamation of the two forms into a single form }\end{array}$ \\
\hline Preference (number of respondents) & $3(10 \%)$ & $13(43.3 \%)$ & $14(46.66 \%)$ \\
\hline
\end{tabular}

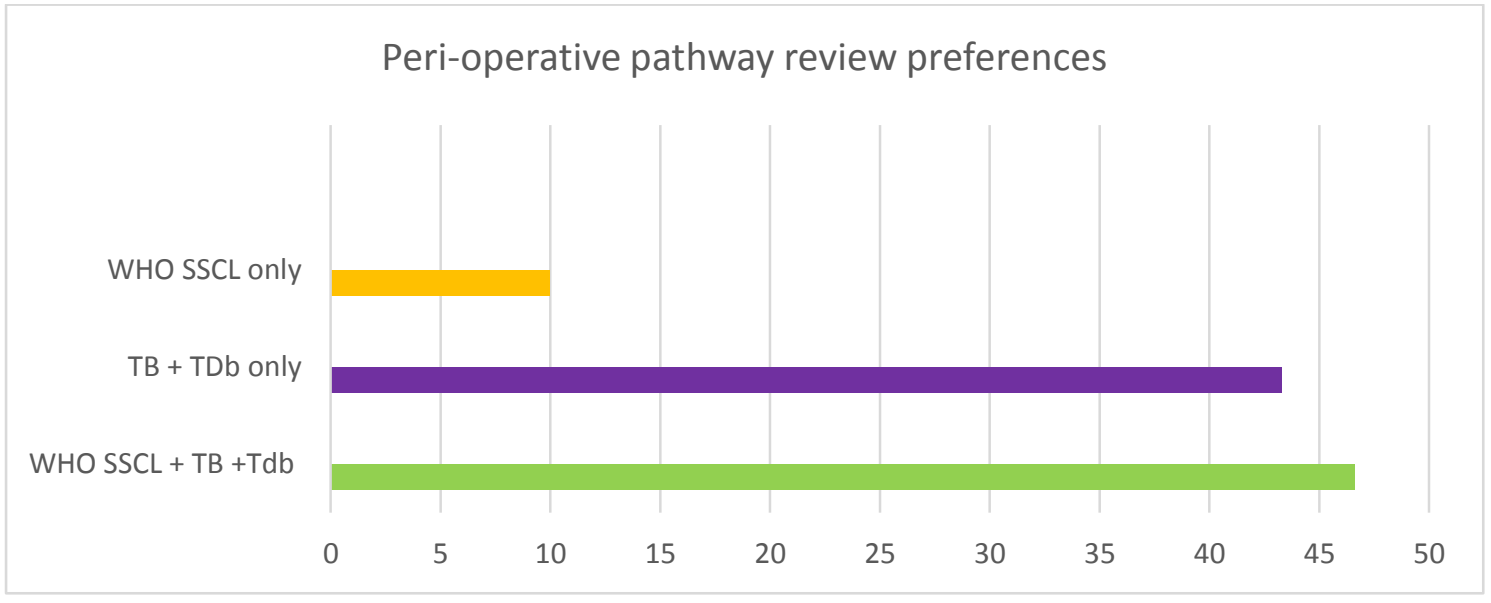

Figure 4: Preferences of respondents for Perioperative safety pathway review 


\section{Discussions}

Standards for Team Brief, WHO - Sign In, Time Out and Sign Out were maintained at close to pre-pandemic (2019) and national standards but there was a $48 \%$ drop in standards with Team Debrief, from pre-pandemic compliance levels of $100 \%$ (DMH 2019 Audits) to $52.0 \%$ in this audit. While it can be argued that the high standards with Team Brief may be related to the near compulsory nature of safety huddles (team brief) have become, the very good compliance with WHO SSCL - Sign In, Time Out and Sign Out cannot also be so easily dismissed, moreover, the fact that team briefs have become compulsory or a generally accepted standard which has been maintained despite the extreme conditions brought on by the pandemic is itself evidence of a true and welcome change in the surgical safety culture in the NHS. The pragmatic use of documentation as proxy for compliance necessitated by the need to avoid intrusions into care processes at a time of increased anxiety is a limitation of this study.

The forty eight percentage point $(48 \%)$ drop in standards with Team Debrief seen in this audit is worrisome because the feedback loop provided by team debriefs for continuous quality and safety improvements is being lost at a time when learning from events is most needed. A number of factors such as fear, fatigue, pains and resource limitations first described by DuPont (1993) in his assessment of aviation incidents may be relevant in the fall in standard seen with Team Debrief. Specific challenges faced by the team and environment studied are revealed in Part B of this study.

The survey of peri-operative staff on COVID-19 anxiety provoking factors (Part B) revealed that cancellation of holidays was rated least of concerns, this may be a pointer to altruism in the healthcare workforce. Also of note is the negative rating of lack or inadequate PPEs, an evidence of significant turnaround in the provisions of PPEs when the pandemic was first declared in the United Kingdom in March 2020 but as many other countries and their healthcare systems still struggle with provisions of PPEs, healthcare workers in such countries may have different experiences from the one reported here. This study also revealed that worries about contracting COVID-19, inadequacies of the current peri-operative care guidelines, difficulties with vision, hearing and physical injuries ("COVID battle scars") when donned with PPEs and work intensity leading to fatigue were rated as high anxiety provoking factors for respondents. With high anxiety levels, staff may be unable or less willing to remain for formal Debriefs after long or difficult surgical procedures. More support with essential resources such as new care pathways, PPEs with better human-system interface, lateral flow (rapid) COVID-19 testing kits and early implementation of mass COVID-19

\section{LMQ Human factors}

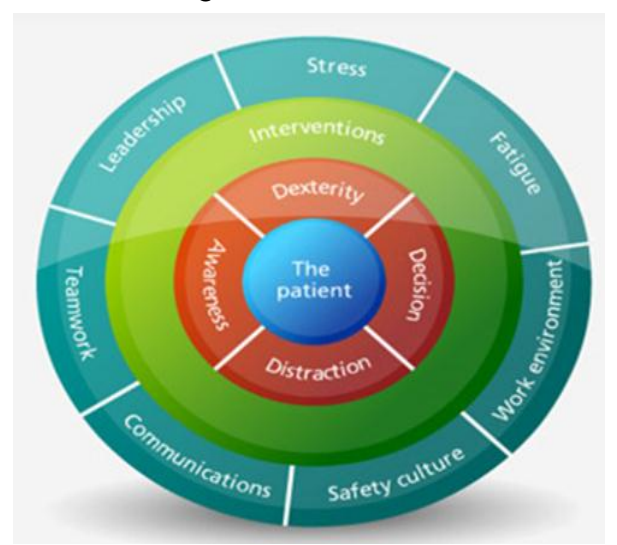

vaccination that have potentials to address the underlying human factors challenges will likely improve compliance and standards because the issues and challenges at play in these findings are traceable to human factors in complex new situations.

Complexity of healthcare safety Management: Surgical safety is complex, even the most straightforward procedures involve dozens of critical steps, each with an opportunity for failure and the potential for injury to patients, from identifying the patient and the operative site correctly, to providing appropriate sterilization of equipment, to following the multiple steps involved in safe administration of anaesthesia, to orchestrating the operation - every step, every resource is critical to success. The most critical resources of operating teams are the knowledge and experience of the constituent clinicians the surgeons, anaesthetists, nurses and operating theatre practitioners, support staff and others, when this multi-disciplinary team work together using their knowledge, abilities (skill mixes) on behalf of the surgical patient, safety is enhanced and considerable proportion of life threatening complications are averted but the effectiveness of even the best teams can be threatened by extreme conditions such as COVID-19 that disrupt teams and corporate wellbeing. To effectively and sustainably manage team effectiveness and staff and patients safety when faced with extreme conditions like the COVID-19, careful analysis of human factors and their management would be essential.

\section{Human Factors: Issues and Challenges}

The central issue of concern with the COVID-19 pandemic in healthcare is the likelihood of higher error rates, therefore, attention to Human Factors/Ergonomics - the study of organisational, individual, environmental, and job characteristics that influence behaviour in ways that can impact safety (chfg.org), will therefore be essential to manage risks and ensure safety.

Risk management is more than safety, "risk" also affects productivity, for example, if the right equipment such as personal protective equipment (PPEs) or the right environment such as optimal operating room temperatures are not available for a hospital surgical team, this will impact not only safety but also theatre productivity as cases will be delayed or cancelled.

Several factors identified in this audit have been known to lead to poor team effectiveness and higher error rates, namely, fear, stress, fatigue and mental load, others are team communication, decision making, situational awareness, information gathering systems, interventions and leadership (DuPont, 2002), (See Figure 5 ), the effective management of these factors require their careful analysis and the pro-active institution of behavioural or performance adjustments to ensure acceptable outcomes (Safety-II) (Hollnagel 2015) (see below).

model Safety-II Approach

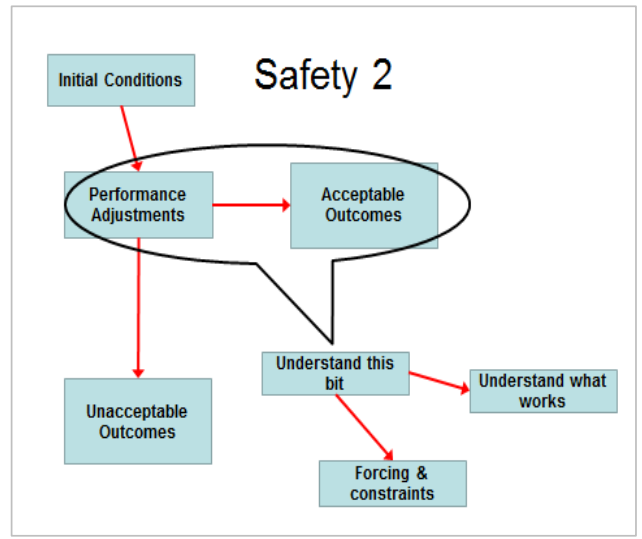

Figure 5: Human factors model. Figure 6: Safety-II: Performance adjustments for acceptable outcomes in all events. 
Human-system interaction is enhanced with the institution of effective human factors tools and techniques, these make teams better adjusted or adapted for optimal performance and productivity, putting the team in a better position to succeed under varying conditions, such as resource and capacity constraints and novel challenging circumstances like the COVID-19 pandemic becoming resilient and reliable, the hallmark of Safety-II (Braithwaite 2015). Resilience enables teams to make the necessary adaptations to ensure good outcomes for familiar and unfamiliar situations (Safety-II).

The ubiquitous nature and severity of the SARS-CoV2, causative agent of COVID-19 pandemic requires a change of strategy from the traditional reactionary response of how things fail -"what has gone wrong" or 'avoiding that something goes wrong' in Safety-I (Patterson 2015) to "ensuring that as many as possible go right' (Hollnagel 2015), that the number of intended and acceptable outcomes is as high as possible - everyday work achieves its objectives and safety management becomes pro-active with regard to how actions succeed in everyday acceptable performance, this means that effective team strategy for the management of COVID-19 in healthcare should focus on analysing potential error sources and developing the necessary behavioural or performance management (human factors) mechanisms to mitigate potential errors.

\section{Human Errors}

An error is the failure of a planned action to be completed as intended (error of execution) or the use of a wrong plan to achieve an aim (error of planning), in other words, the failure of a planned action to achieve its intended outcome or a deviation between what was actually done and what should have been done or doing the wrong thing when meaning to do the right (Reason, 1990). Several types of errors or unsafe acts exist (Reason, 1990).

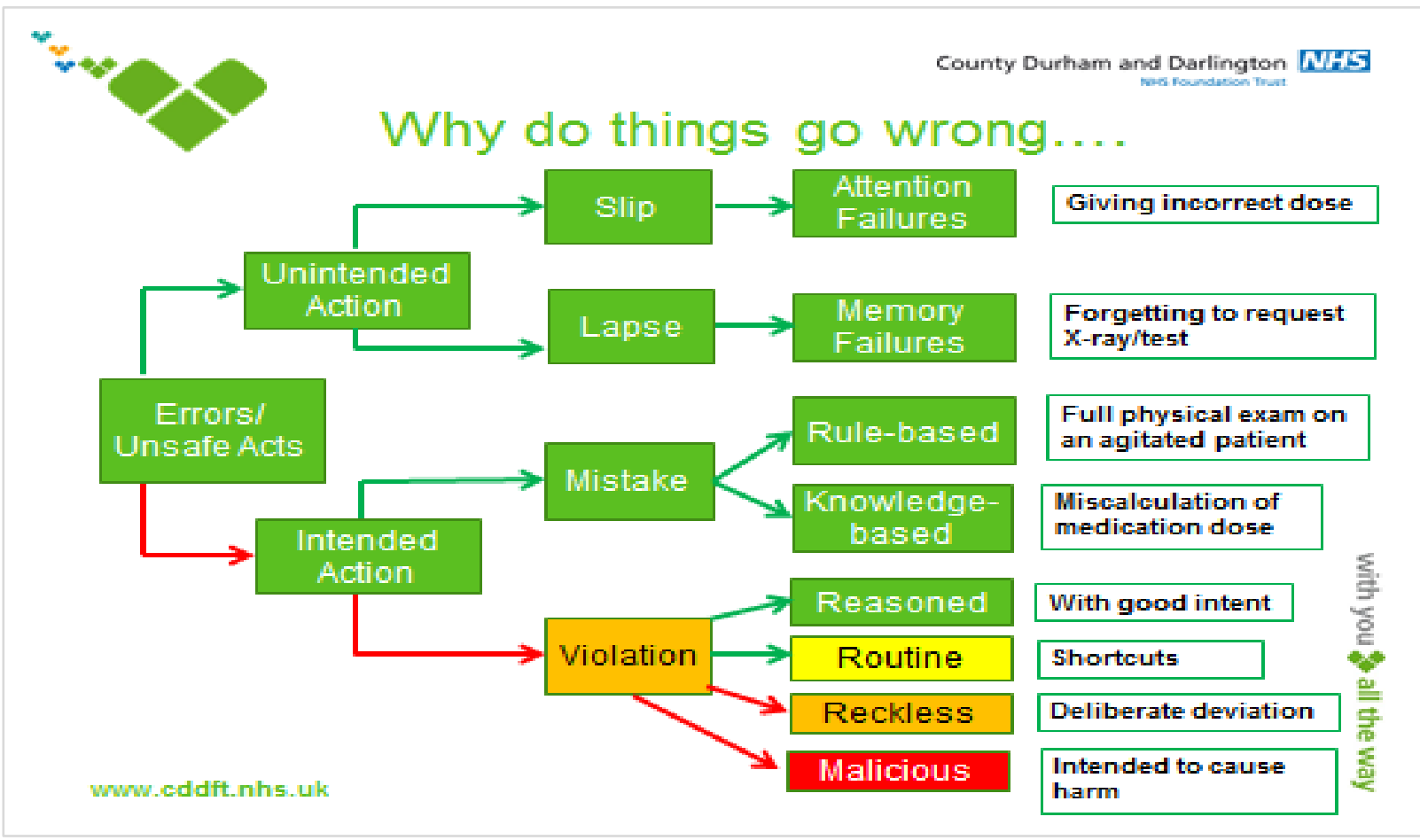

Human error classification (Reason, 1990) modified by NPSA for healthcare (RCA).

James Reason classified human errors in 1990 based on observations from organisations that have become highly reliable organisations (HRO), industries such as aviation and nuclear power. In this classification, errors arise for two reasons: active failures and latent conditions, active failures are unsafe acts committed by people who are in direct contact with patients; these are intended and unintended actions occurring in a variety of forms such as slips, lapses, and mistakes and violations.

\section{Intended and Unintended Actions}

Resource constraint, an inevitable consequence of the COVID-19 pandemic in healthcare, together with stress, fear, fatigue, physical injuries, visual and hearing impairments when PPEs are donned have the potential to trigger both intentional and unintentional actions in care processes involving slips, lapses, mistakes and violations (Reason, 1990). It is noteworthy that in managing extreme conditions such as COVID-19, deliberate acts of violations will occur, but in safety conscious organisations, these will be reasoned (considered and discussed) violations rather than reckless or malicious violations, examples of this (reasoned violations) include the use of anaesthetic ventilators (safe but not licensed for prolonged ICU ventilation) for ICU patients during the pandemic and the use of Operating theatres for ICU care. Similarly, to meet the challenge of acute manpower shortages, non-ICU nurses were given short induction followed by on the job support to care for ICU patients and retired clinicians called to return to frontline care.

The increased workload for healthcare staff brought on by the COVID-19 pandemic that led to calls for retired professionals to re-join active workforce and the use of non-critical care staff for critical care roles, have safety risks from unfamiliarity with tasks, inexperience, reduced quality of supervision - all these can potentially increase harm to staff and patients through higher error rates. Individual factors that predispose to errors include inexperience (those returning to care from retirement as described above, trainees and juniors), fatigue, stress, hunger, illness and cultural factors (language barrier), all these can further increase the risk of patient harm (Vincent et al 1993).

Fear Factor: Fear leading to higher rates of anxiety, sicknesses and absences has become a major challenge in the healthcare during the COVID -19 pandemic. Fear alters the physiological processing of impulses, when sensory impulses are perceived to be 
threatening, the processing changes from conscious (reasoned approach) normally through the cortex to a more rapid alternative pathway through the amygdala (intuitive route), when the amygdala receives sensory impulses that are perceived to be threatening and close to the object (human), a rapid reaction (response) which may not be reasoned or rational is discharged.

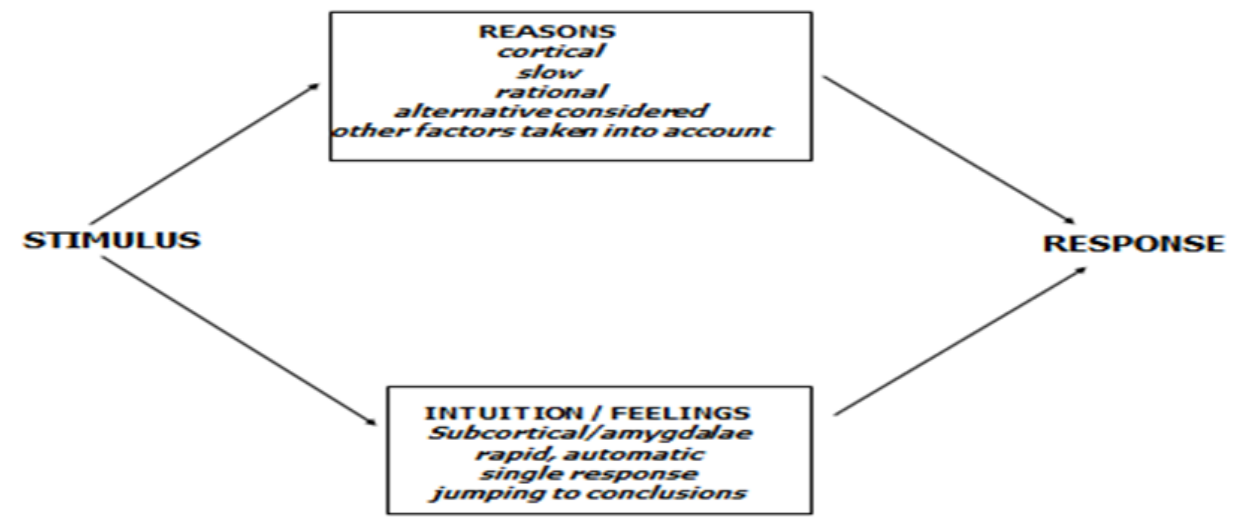

With COVID-19 induced fear and anxieties, there is concern that healthcare stimuli-responses processing will engage the intuitive/feelings route more frequently compared to normal times with the risk of higher error rates. Management includes the use of checklists for cognitive offloading and prevention of automatic actions.

Fatigue: 24 hours of sleep deprivation has performance effects equivalent to blood alcohol content of $0.1 \%$ - disturbances in reasoning, depth perception, peripheral vision, possibility of nausea and vomiting, reflexes (reaction time), gross motor control, staggering, and slurred speech (Dawson and Reid, 1997). The European Working Time Directive (2007) that specified the maximum continuous working periods for healthcare staff was aimed at preventing these risks of fatigue in healthcare, recently, the advice from the Clinical Human Factors Group (chfg, 2020) for the establishment of resilience rotas which provide for staff backups - colleagues placed on standby to step in when anyone on duty is exhausted or sick is an excellent strategy for improving team resilience.

Stress and Performance: The optimum level of performance is reached when the level of arousal is neither too high nor too low (Yerkes and Dodson, 1908). Boredom is a problem if we are doing a highly automated and repetitive task, likewise, when involved in a prolonged high stress situation performance diminishes as fatigue sets in.

\section{Stress and performance}

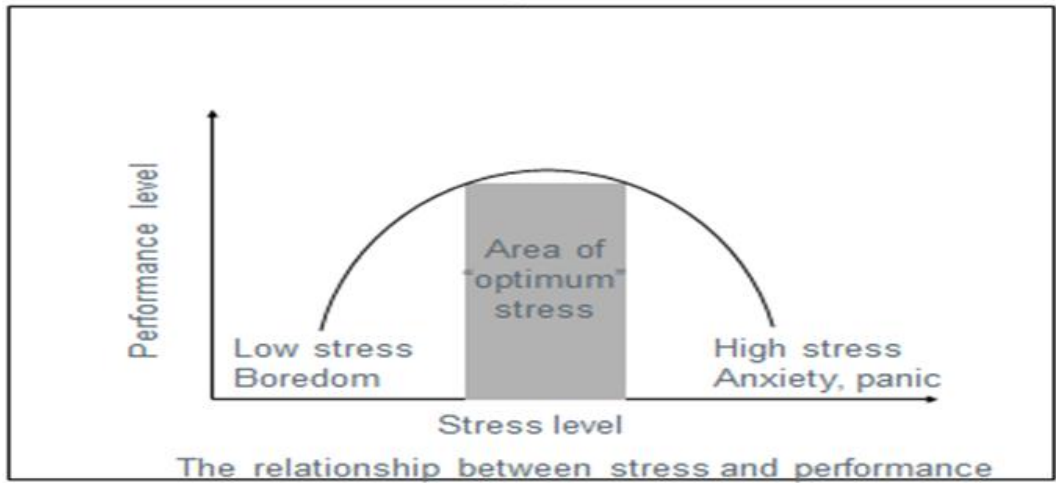

(Yerkes, \& Dodson, 1908)

Managing Risks in Healthcare: The effective management of the risks discussed above require the institution of strategies in the form of persons, tools and technology to ensure that procedures are safe and teams achieve their goals despite changes in prevailing circumstances (Safety-II). Against the sobering statistics of unmet needs in healthcare that existed pre-pandemic (WHO, 2000), this alternative (Safety-II) approach to safety management that equip teams and organisations to succeed in varying circumstances would be an essential or critical success factor in the delivery of safe care. Safety-II approach is deployed in healthcare through human factors science, tools and techniques.
Human Factors science, tools and techniques: Human factors science acknowledges the universal nature of human fallibility and the inevitability of error, it also assumes that errors will occur and therefore focusses on designing processes and interactions to minimize the likelihood of errors or its consequences. An example of human factors model for managing design processes and humansystems interactions is the SHEEP (Systems, Human interaction, Environment, Equipment and Personnel) model: Systems - how information flows, how it is stored and transferred, Human interaction - team leadership/followership, approachability and assertiveness, emotional intelligence for self-awareness and selfmanagement, teamwork, situational awareness, decision-making, 
task management and information transfer. Environment conditions affecting physiological responses such as temperature, noise levels (decibels); planning and layout of areas and moving from one environment to another. Equipment - machines, consumables and non-consumables, equipment storage, maintenance, standardization. Personal - personal role, adequate intake (food and water), fatigue, stress, significant life events such as divorce, illness or supporting others (Rosenorn-Lanng, 2014). Another example of a human factors tool is the PETTO (People, Environment, Tools and Technology, Tasks, processes and Organisations.

Judicious application of these human factors tools help teams to standardise and simplify tasks, procedures and interactions for the reduction of risks and improvement of safety.

In practice, human factors science, tools and techniques are embedded into operational templates such as: Task management, Team working, Situational awareness, Decision making, Leadership - this should be collaborative, open, transparent and democratic, as the culture of openness makes feedbacks easier to give for the review of old goals and the setting of new ones (Dekker and Hugh, 2010) and Effective communication - the glue that keeps teams together, helping to improve knowledge and shared understanding of new threats such as concerns over "collateral damage" caused by the "unjustifiable use" of scarce resources on COVID-19 patients to the detriment of other patients (Loannidis, 2020) and the nature and timing of appropriate responses to such threats to ensure safety for all concerned.

\section{Conclusions}

The extreme circumstances and disruptive human factors brought into healthcare by the COVID-19 pandemic have impacted care processes, standards and the safety of staff and patients. Building team and organisational resilience through effective communication and support with essential resources such as new care pathways, PPEs with better human-system interface, lateral flow (rapid) COVID-19 testing kits and early implementation of mass COVID-19 vaccination would be key to change - a practical demonstration of a paradigm shift in the approach to safety management from the traditional Safety-I approach of "avoidance" to Safety-II that focusses on building teams and organisational resilience to succeed in varying circumstances.

\section{References}

[1] Allard J, Bleakley A, Hobbs A, et al. (2011) Pre-surgery briefings and safety climate in the operating theatre. BMJ Qual Saf 20(8): 711-7.

[2] Brady, P, Goldenhar, L (2014) A qualitative study examining the influences on situation awareness and the identification, mitigation and escalation of recognised patient risk BMJ Quality and Safety 23 (2) 153-161.

[3] Braithwaite J, Wears RL, Hollnagel E. Resilient health care: turning patient safety on its head. Int J Qual Health Care. 2015;27(5):418-420. doi: 10.1093/intqhc/mzv063. [PubMed] [CrossRef] [Google Scholar]

[4] Classen DC, Evans RS, Pestotnik SL, Horn SD, Menlove RL, Burke JP. The timing of prophylactic administration of antibiotics and the risk of surgical-wound infection. $\mathrm{N}$ Engl J Med 1992;326:281-6.

[5] Care Quality Commission (2017) Inspection framework: NHS acute hospitals Care Quality Commission Available from: https://www.cqc.org.uk/sites/default/files/20171219_nhscore-services-framework-acute_surgery.pdf (Accessed 22 July 2020)

[6] Clinical Human Factors Group (2020). Key Human Factors messages when working under pressure: https://chfg.org/category/all-resources/covid-19/ Apr 16, 2020. Accessed April 16, 2020

[7] Clinical Human Factors Group (2020). https://chfg.org/what-are-clinical-human-factors/ Apr 16, 2020 Accessed 28 April 2020

[8] Dawson, D., Reid, K. (1997). Fatigue, alcohol and performance impairment Nature 388: 235

[9] Dekker, S.W.A., Hugh T.B. (2010). Balancing "no blame" with accountability in patient safety. NEJM, 362 (3):275.

[10] DuPont, G., (1993). Human Performance Factors for Elementary Work and Servicing. Transport Canada TC14175.

http://www.skybrary.aero/bookshelf/books/2038.pdf [Accessed 25 August 2020].

[11] Einav Y, Gopher D, Kara I, et al. (2010). Preoperative briefing in the operating room: Shared cognition, teamwork, and patient safety. Chest 137(2): 443-9.

[12] Greer M, Curdy N, Kopolow A, Mercado SE. Competencies for patient safety and quality improvement. Jt Comm J Qual Patient Saf. 2016;42(10):479. doi: 10.1016/S1553-7250(16)42087-8. [CrossRef] [Google Scholar]

[13] Haynes A, Weiser TG, Berry WR, et al. (2009). A surgical safety checklist to reduce morbidity and mortality in a global population. New England Journal of Medicine 360: 491-9.

[14] Hollnagel E, Wears RL, Braithwaite J. From Safety-I to Safety-II: A white paper. Published simultaneously by the University of Southern Denmark, University of Florida, USA, and Macquarie University, Australia: The Resilient Health Care Net; 2015. http://resilienthealthcare.net/onewebmedia/WhitePaperFi nal.pdf. Published 2015. Accessed 28 April 2020.

[15] Hui DS, I Azhar E, Madani TA, Ntoumi F, Kock R, Dar O, et al. (February 2020). "The continuing 2019-nCoV epidemic threat of novel coronaviruses to global healthThe latest 2019 novel coronavirus outbreak in Wuhan, China". Int J Infect Dis. 91: 264-66. doi:10.1016/j.ijid.2020.01.009. PMC 7128332. PMID 31953166.

[16] Institute of Medicine (2000). To Err is Human: Building a Safer Health System. Institute of Medicine (US) Committee on Quality of Health Care in America; Kohn LT, Corrigan JM, Donaldson MS, editors. Washington (DC): National Academies Press (US); 2000.

[17] Jain AL, Jones KC, Simon J, et al. (2015). The impact of a daily pre-operative surgical huddle on interruptions, delays, and surgeon satisfaction in an orthopedic operating room: A prospective study. Patient Safety in Surgery 9: 8. Leape, LL. (1994) Errors in Medicine, JAMA; 272 (23): 1851-57

[18] Leonard M, Graham S, Bonacum D. (2004). The human factor: the critical importance of effective teamwork and communication in providing safe care. Quality and Safety in Healthcare 13: 185-90.

[19] Lingard, L, Regehr, G, Carmill, C, et al (2011) Evaluation of a preoperative Team Briefing: a new 
communication routine results in improved clinical practice BMJ Quality \& Safety 20 (6) 475-482

[20] Loannidis, JPA (2020): Coronavirus disease 2019: the harms of exaggerated information and non-evidencebased measures. Eur J Clin Invest. 2020 Mar 23:e13223. doi: 10.1111/eci.13223.

[21] National Patient Safety Agency (2010). Five steps to safer surgery, London, NPSA.

[22] National Health Service Institute for Innovation and Improvement. 2009. Saving lives in surgery: A guide for chief executives in implementing the surgical safety checklist.

[23] Patel J, Ahmed K, Guru KA, et al. (2014). An overview of the use and implementation of checklists in surgical specialities: A systematic review. International Journal of Surgery 12(12): 1317-23.

[24] Patterson M, Deutsch ES. Safety-I, Safety-II and resilience engineering. Curr Probl Pediatr Adolesc Health Care. 2015;45(12):382-389. doi: 10.1016/J.CPPEDS.2015.10.001. [PubMed] [CrossRef] [Google Scholar]

[25] Papaspyros SC, Javangula KC, Adluri RK, et al. (2010). Briefing and debriefing in the cardiac operating room. Analysis of impact on theatre team attitude and patient safety. Interact Cardiovasc Thorac Surg 10: 43-47, 201.

[26] Public Health England (2020). https://www.gov.uk/government/collections/coronaviruscovid-19-list-of-guidance\#guidance-for-healthprofessionals Apr 8, 2020 Accessed April 16, 2020

[27] Sexton JB, Makary MA, Tersigni AR,et al. Teamwork in the operating room: frontline perspectives among hospitals and operating room personnel. Anesthesiology 2006;105:877-84.

[28] Spurgeon, P., Cross, S. (2020). Patient Safety Syllabus 1.0 Academy of Medical Royal Colleges, London.

[29] Reason, J. (1990). Human Error, Cambridge University Press, Cambridge. Cambridge, MA.
[30] Reason, J. (1995). "A System Approach to Organizational Error". Ergonomics. 38: 1708-1721. doi:10.1080/00140139508925221.

[31] Rosenorn-Lanng, D. (2014). Human Factors in Health Care. Oxford University Press Clays Ltd, St Ives. Treadwell J, Lucas S, Tsou A. (2014). Surgical checklists: A systematic review of impacts and implementation. BMJ Quality Safety 23(4): 299

[32] Vickers, R (2011) Five steps to safer surgery Annals of Royal College of Surgeons for England 93 (7) 501-503

[33] Vincent, C, Ennis, M, Audley, R. (1993). Medical Accidents, Oxford Medical Publications, Oxford. Whitty C. (2020). Need for Continuous Quality Improvements during the COVID-19 pandemic. Available at www.nihr.ac.uk/covid-19/[Accessed 30th March 2020].

[34] World Health Organization (2000). World health systems: Improving performance, Geneva, World HealthGuidelines for Safe Surgery (2009): Safe Surgery Saves Lives. Geneva: World Health Organization.

[35] World Health Organization (2008). The Second Global Patient Safety Challenge 2007-2008: "Safe Surgery Saves Lives" Geneva, World Health Organization.

[36] WHO guidelines for safe surgery (2009): safe surgery saves lives. Geneva: World Health Organization; 2009 (http://apps.who.int/iris/bitstream/handle/10665/44185/9 789241598552_eng.pdf?sequence $=1$ [Accessed 20th July 2020].

[37] World Health Organization (2020). World Patient Safety Day 2020: Staff Safety: A priority for Patient Safety www.who.it/wha72.6/2019 [accessed 17th September, 2020].

[38] Yerkes, R. M., Dodson, J. D. (1908). The relation of strength of stimulus to rapidity of habit-formation. Journal of Comparative Neurology and Psychology, 18, 459-482. 